\title{
SKIN: An Open Negotiation Between Form, Function and Materiality
}

\author{
Gernot Riether \\ Ball State University \\ griether@bsu.edu \\ Keyan Rahimzadeh \\ Georgia Institute of Technology \\ keyanr@gmail.com
}

\begin{abstract}
The Nuit Blanche Pavilion is a recent project by Gernot Riether in collaboration with artist Damien Valero. The project takes advantage of an elastic behavior found in high-density elastomers to form a lightweight structure that integrates form, geometry and program in a single material system. Grasshopper and the finite element plug-in Karamba were used to optimize these interdependencies. This paper will describe the design of the project and elaborate on an aspect of Riether's research that uses digital technology to promote environmentally responsible materials and construction methods.
\end{abstract}

Keywords: Parametric design; Polymers; Light weight structure; Interactive video installation.

\section{Introduction}

Comparing construction systems commonly used in the building industry to natural systems, one soon realizes that in nature the interdependency between structure, geometry, material, appearance and environmental influences is far more complex than in buildings. Geometry for instance might emerge as a consequence of an open negotiation between material properties, function and environmental influences. Architectural design tools that combine parameterized geometric models with finite element calculation and optimization algorithms allow us to get a bit closer to the way nature "designs." Inspired by nature the goal of the research is to develop a new generation of environmentally responsible building systems that are more efficient in saving

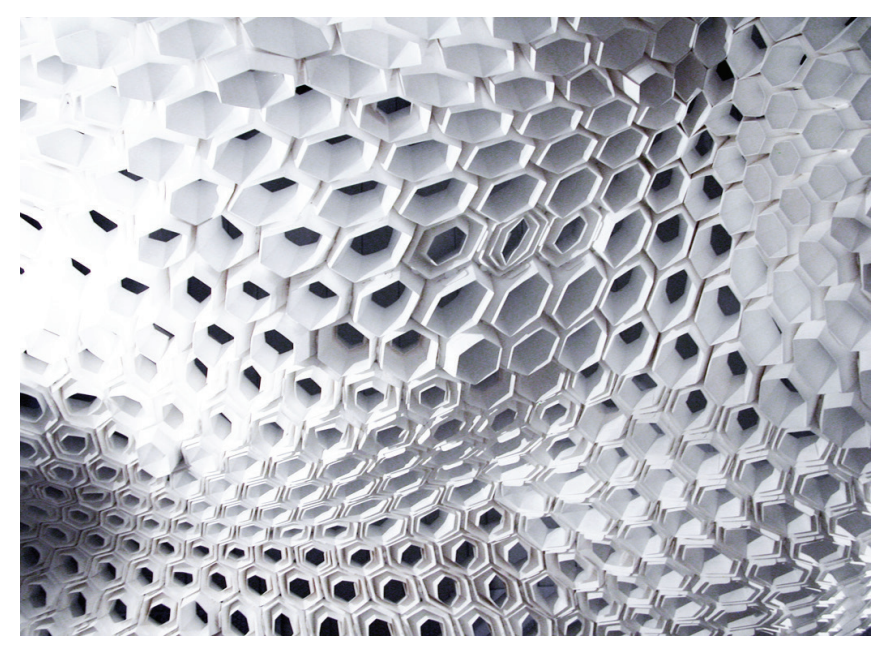

Figure 1: Detail of Coded Surfaces, Rome, Italy, June 2010. material and responding to environmental conditions in a more intense and dynamic way.

This thinking has informed a body of work that we have developed over the past three years. All of the projects are developed from modular-based systems, calling attention to the material properties of environmentally friendly polymers. Projects, such as the Coded Surfaces installation in Rome for instance used the variation of folds in the module to inform the curvature of larger surfaces. Increasing the complexity of projects to freestanding pavilions allowed us to integrate more parameters in a generative design process and increase the complexity of the solution. The AIA Pavilion and the MainX24 Pavilion were an attempt to synthesize material behavior with digital design and fabrication techniques. Properties that are typically ignored in the conventional use by the building industry, such as the elasticity of polymers, thereby became essential drivers in our design process. However the value of the research in the pavilion extends beyond digital design or fabrication technique and material exploration to encompass functions, site conditions and other aspects of design. For this reason, we see the scale of the pavilion as an opportunity to test and experiment with generative design methods that allow us to respond to the complexity of context and material in new ways.

\section{Function}

The project began with an invitation of the Cultural Ministry of Paris to design a pavilion for Nuit Blanche 2012, an annual art festival in Paris, France. The pavilion had to serve as an 
infrastructure for an interactive art installation by artist Damien Valero. The design of the pavilion responded to Valero's investigations in human skin as organic interface. The pavilion's overall from responded to a video installation and the requirements of a space that allowed specific cones of vision.

Valero's work is interactive in nature and is characterized by an extensive use of digital media, which challenged the convention of a typical art display or gallery space. In addition, the goal was to build the pavilion as a deployable structure that might be used for other events to come. It was further required that the pavilion allow for easy transport and assembly without large equipment.

The main idea for the pavilion was to develop a double skin system that would form the structure of the pavilion and be adaptable for different interactive art installations. To make the pavilion deployable the envelope was divided into modules, each module consisting of two halves. Each of the modules could be programmed differently based on the needs of the individual installation and could function as container for sensors, small video projectors or other devices needed for different installations. Since the two parts of each module were connected through bolts along the aperture, each module could be opened and closed to access the module's interior at any time after the pavilion was assembled. The continuity of the double-skin system on the other hand provided an opportunity to run an infrastructure of cables connecting the different modules. Sensors and display devices could therefore easily be networked by opening and closing different modules after the pavilion was completed.
Base geometry:

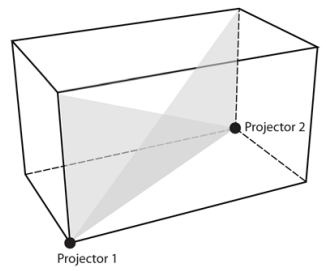

Merging the surface in the middle:

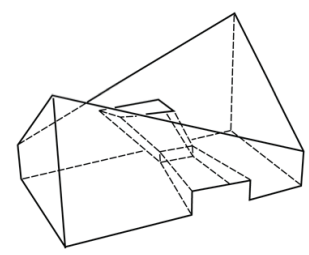

Compressed corners:

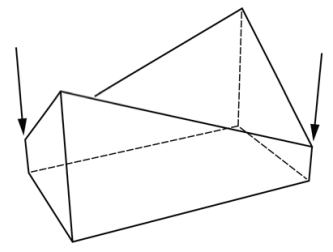

Softening geometry:

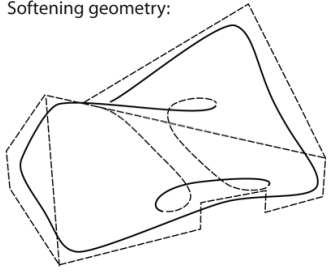

Figure 2: The form was optimized for cones of vision, necessary for the video installation.
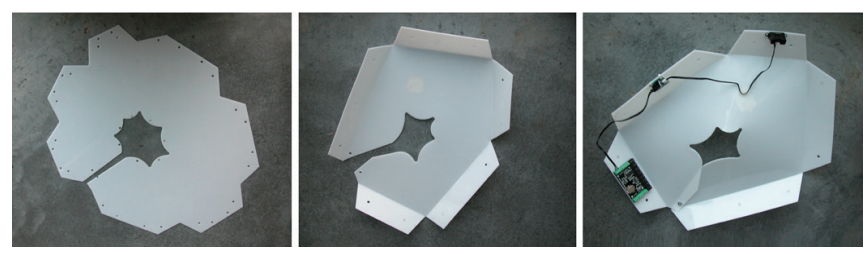

Figure 3: Module part with integrated motion sensor.

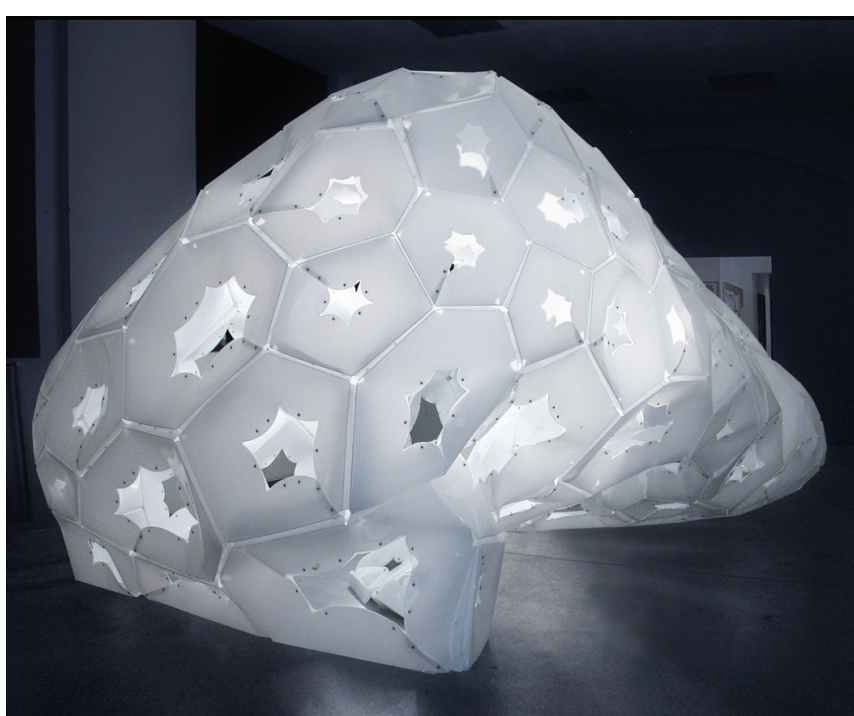

Figure 4: Overall view of the pavilion.

\section{Elastomers}

As the chemical industry changes from fossil fuel based plastics to bio plastics, our research focuses on developing techniques and methods that reintroduce plastics as a building material in a new form. In doing so, we are also suggesting new spatial and aesthetic qualities that are essential to changing the perception of plastic from an environmentally problematic to an environmentally friendly material.

In her New York Times article "Plastic: Too Good to Throw Away," Susan Freinkel notes, "The problem isn't the plastic, but the way we use it." She argues that plastics are too valuable to be wasted on cheap junk, but should be reserved for the things that can best take advantage of its properties. We have experimented for a while with possibilities for introducing recycled plastics and bioplastics into architecture. Of all the plastics, it is ETFE, ethylene tetrafluoroethylene in particular that is currently enjoying a revival in architecture. However, a closer look reveals that this material only informs a very few types of architectural systems, such as pillow systems.

The Nuit Blanche Pavilion is part of a research agenda that explores elastomers as a potential building material. The project is a part of an ongoing research on the behavior of a large range of available elastomers as potential materials for structural envelopes, as we have described in papers presented at eCAADe 2012 and ACADIA 2011.The Nuit Blanche Pavilion extends these explorations by taking advantage of the material properties of high-density polymers. Such polymers have much stronger intermolecular forces than regular polymers resulting in an increase in material strength. 


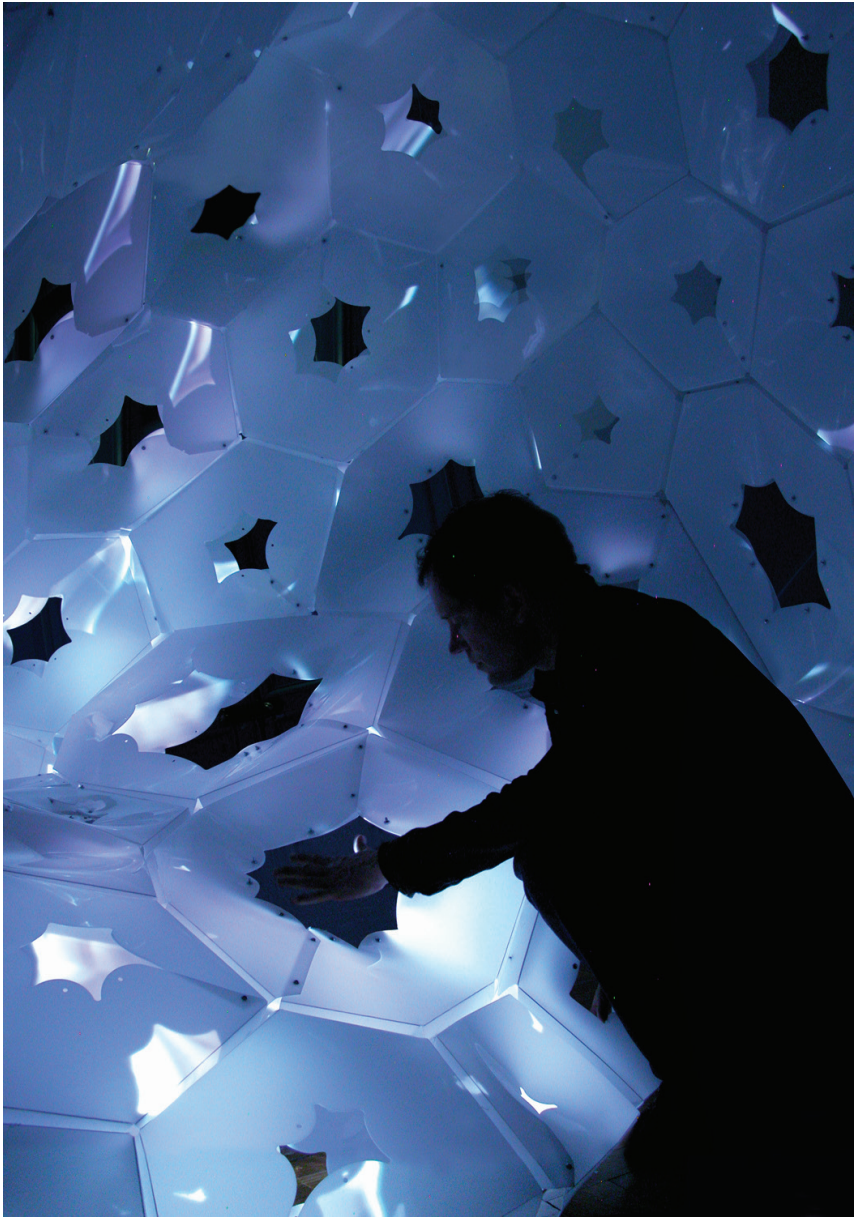

Figure 5: Nuit Blanche Pavilion, interactive lighting.

Depending on the resin type, reinforcing agents, and processing methods, the material performance in tension and compression can vary from one another by $50 \%$ or more. This differs from many conventional building materials, such as metals, where the tensile and compressive moduli are very close. In distinction from most building materials, the behavior of polymers can be heavily manipulated through different processing methods and aggregates. The behavior of elastomers can be changed and adapted more easier than most materials commonly used in the building industry.

Because polymers are easy to recycle, they are increasingly used for bottles and packaging. Polymers have also benefitted from evaluations of possible health and environmental problems caused by PV and Polycarbonates, as well as from environmental advantages over glass, metal and cardboard. The industry is in the process of changing its production to bio-polymers that are based on starch, sugar, cellulose or synthetic materials. Though its original production is petroleum-based, the material we used is fully recyclable. The larger goal of this research is to inspire or develop new building systems that will benefit from the environmental advantages of bio-plastics.

\section{Structure}
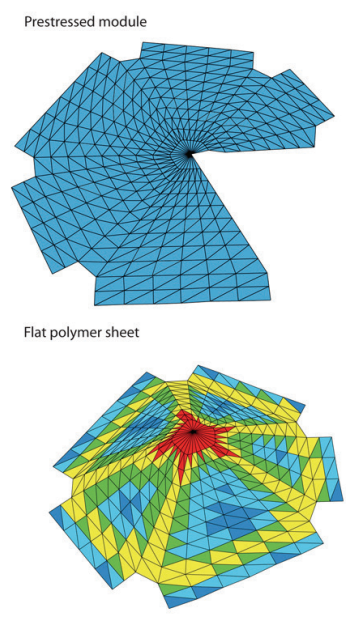

Sheet foldend into a cone shape

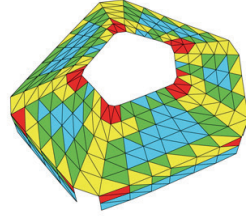

Module half with removed material from the cente.

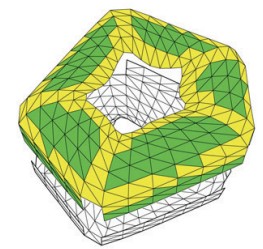

Module halves connected to each other after after beeing assembled

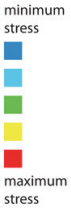

Figure 6: Stress simulation of module.

The design of the Nuit Blanche Pavilion started by testing the behavior of different high-density polymers. A series of studies tested possibilities to shape the material and experiment with different means of connections. Polymers are usually formed into final shapes through vacuum forming, draping and drape forming. Instead of using only the material's malleability, this project also tested techniques using its elastic qualities. In one of our previous projects, the AIA Pavilion in New Orleans we pre-stressed a surface by bending the envelope as a whole and pinning it to the ground. At the Nuit Blanche Pavilion we were interested in prestressing smaller sections of the surface that would support each other. The strategy of stressing and connecting a series of individual sections has also been used by Achim Menges in his Bending-Active Pavilion. But instead of dividing the pavilion in cross sections as done in the Bending-Active Pavilion we wanted to build up tension by varying relationships between the different modules of a shell structure. This was achieved by developing a module in two parts that, once connected to each other, formed a double-layered surface. This allowed us to use the elasticity of the material in two ways: first to develop rigidity within the module and second to create stiffness in the edges between the modules.

\section{Geometry}

We investigated different base geometries for the cell in relation to the overall form, such as different triangulations and Voronoi patterns. From Buckminster Fuller's dome, we know that altering hexagons and pentagons in two directions is the most structurally efficient way to generate a sphere. A single curvature therefore is a result of altering hexagons and pentagons in one direction, as in nanotubes. We concluded that optimizing a surface that changes from a double to a single curvature is therefore likely to result in different distributions of geometries. Buckminster Fuller's dome uses a geometric principal to optimize a structural system that can also be found in nature in molecules composed of carbon. We 
were looking for other examples in nature that use more complex geometrical patterns to optimize structure.

We found that the structure of the exoskeleton of beetles uses Voronoi pattern that changes in its formal complexity. The green beetle, Chrysina Gloriosa, possesses an exoskeleton that is made up of cells $(\sim 10 \mathrm{~mm})$ that combine pentagons and hexagons. Figure 7 shows a Voronoi analysis of an optical micrograph of the exoskeleton of a beetle. The diagram to the right shows a decrease in the fraction of hexagons in the pattern as a consequence of an increase in curvature.
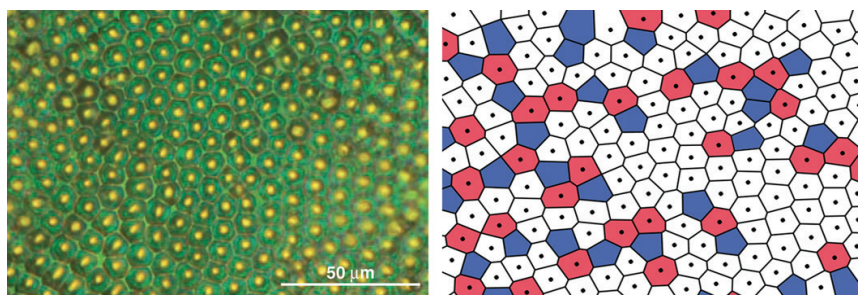

Figure 7: Voronoi pattern of green beetle, Chrysina Gloriosa.

These same principals were adapted for the pavilion. We tessellated the surface into a cellular system of altering hexagons and pentagons. The final distortions and distributions of pentagons and hexagons resulted from a negotiation between formal, functional and structural relationships. Rhino, RhinoScript and Grasshopper and Karamba was used to synthesize these forces in a single system that could simultaneously respond to a wide range of attributes. We first related the design of the module to the overall form that was derived from spatial requirements of the program.
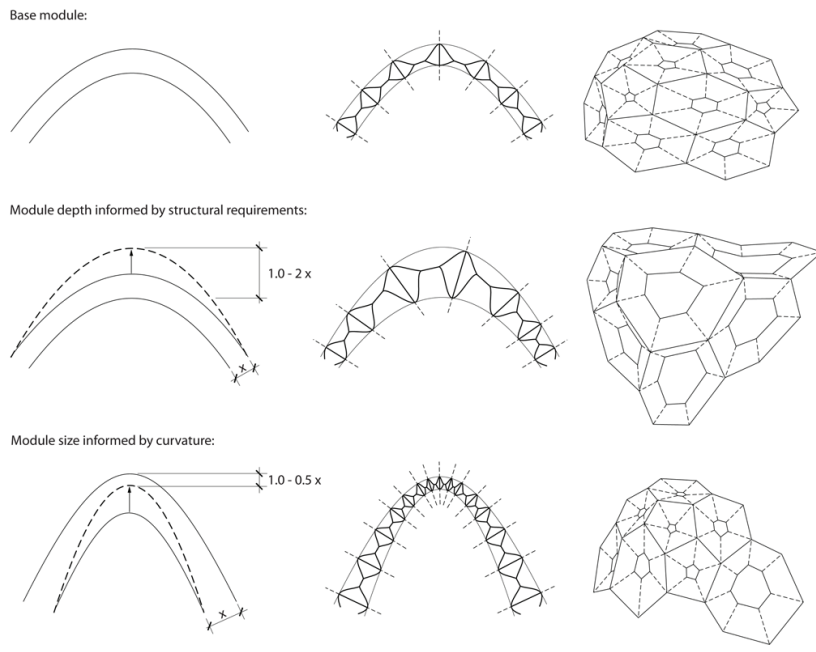

Figure 8: Relation between curvature and module.

The size of the modules was determined relative to the shape of the surface area and the size of modules was determined relative the depth of the envelope. We then related the design of the module to functional requirements. The envelope had to accommodate doors and windows. The required size of different types of apertures was also related to curvature. A different set of requirements dealt with the infrastructure of the interactive art installations such as sizes and locations of sensors and display devices. A minimum depth and a minimum and maximum size of each module were determined by the spatial requirements of installation equipment.

\section{Integration and optimization of structure and geometry}

This geometric system was then optimized for the structural characteristics of ETFE. Individual modules acted as plate elements that are connected to form a larger structure. Because plate behavior is predicated on the continuity of material, removing material from the plate's center suggested the structure to be capable of gradually changing its behavior from a shell structure to a wireframe structure consisting of linear frame elements. The two domains, wireframe and shell structure, were used as guidelines to perform different analytical methods with an understanding that the actual behavior would be in an ambiguous domain between the two.
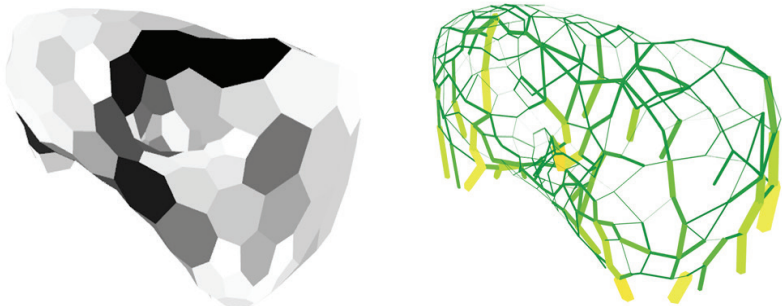

Figure 9: Structural analyses: Left as shell structure, right as wireframe.

In modeling the pre-tensioned modules we investigated two aspects of plate behavior: in-plane and out-of-plane stresses. The in-plane stresses are the results of compression and tension forces, and the out-of plane stresses correlate to moment loads. Removing the material in the center of the module allowed us to optimize the pavilion on the global scale by shifting the in-plane forces to the edge condition, where the addition of flanges and the doubling of plates increased stiffness and capacity. This had the adverse affect of also increasing the risk of buckling in those plates. However, the pre-stressing that resulted from the convex warping of the modules as they assembled stiffened the modules against out-of-plane buckling. Thus, reducing the material in the center of the module, shifting load transfer to module edges, and reinforcing against out-of-plane buckling validated a wireframe model as an approximation. Analyzing the model as a wireframe created an analogue to forces that you would find in linear members: In-plane relating to axial forces, and out-of-plane to moment and bending forces. This provided us with sufficient information that we could use to feed back into the model to 
optimize the design of the module. The first method was through iterative deformation compensation. Using the finite element plug-in Karamba for Grasshopper, the structure was analyzed as a wireframe, with the edges of the modules comprising the 1-d finite elements. Deformations were calculated using static analysis. The current distribution and length of nodes (module edges) compromised the ideal distribution of stiffness and flexibility of the envelope. To relate the geometry to structure an optimization algorithm was developed that moved each node along the envelope in relation to its 8 closest neighbors to guarantee a minimum in stiffness and maximum in flexibility. The algorithm steps through each node, locates its nearest neighbors, determines which node experienced the most deformation under the static load, and then moves toward the node, travelling a distance proportional to the amount of deformation. This locally reinforces whichever nodes are deflecting the most, thus introducing more edges and thereby more stiffness. The original topology is maintained until the regularity of the polygon faces is lost, at which point a new Voronoi pattern can be determined.

Other optimization methods were pursued that chose geometry as a driver, with structural considerations as a result. The first method took the original Delauney mesh that was the dual of the Voronoi pattern used, and evenly distributed its nodes across the surface, where each node was moved iteratively based on the square of its distance to its nearest neighbors. Upon redistribution, a new Voronoi pattern was derived with a completely unique connectivity. This same notion was applied to the vertices of the original Voronoi design - redistributing its vertices across the surface, then rebuilding polygonal faces. Thirdly, a separate algorithm distributed nodes base on the equalization of edge lengths. In this case, the original connectivity was maintained, but the modules had more similar areas.

\section{Fabrication and assembly}

After the cells were generated in Grasshopper, the 640 parts of the 320 individual cells were numbered. To track the final orientation of each cell the edges had to be labeled as well. The cells were then unfolded and all the connection details were added to the unfolded geometry. The final geometries were nested on 4' $\times 8^{\prime}$ sheets of a high-density polymer. CNC was used to cut all 640 outlines of the pavilion's cells. To make the assembly process more efficient, pieces were grouped in topographical layers that reflected the sequence of the assembly process.

Putting the numbers of pieces as well as edge numbers in the sequence of the assembly process allowed for a completely selfguided assembly process that didn't require any additional diagrams or drawings. It was therefore possible to ship all the pieces of the pavilion from the United States to Paris and engage a local team of students to assemble the pavilion.

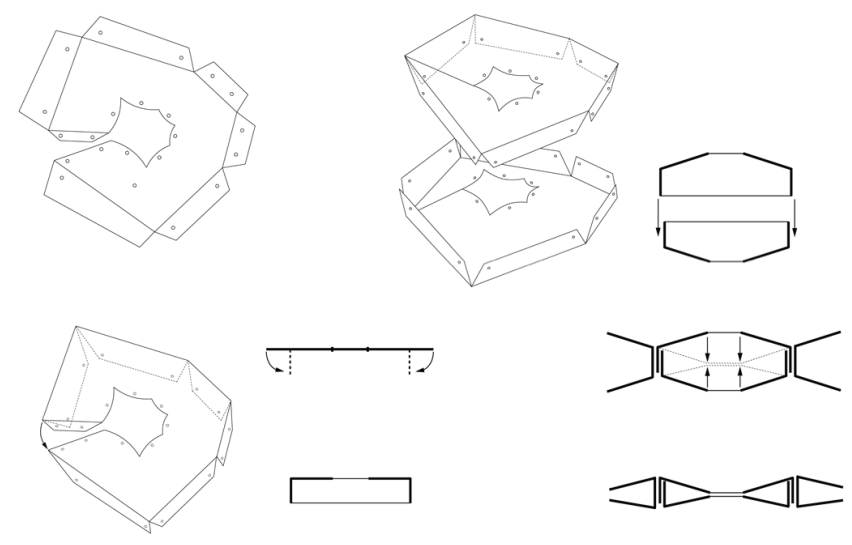

Figure 10: Assembly of module.

The entire assembly by a team of six people took less then two days. The research of the beetle's exoskeleton was further adapted by artist Damien Valero to design the video installation for the pavilion. The Voronoi texture of the beetle's exoskeleton not only provided the basis for the morphogenesis of the exoskeleton but also created its color.

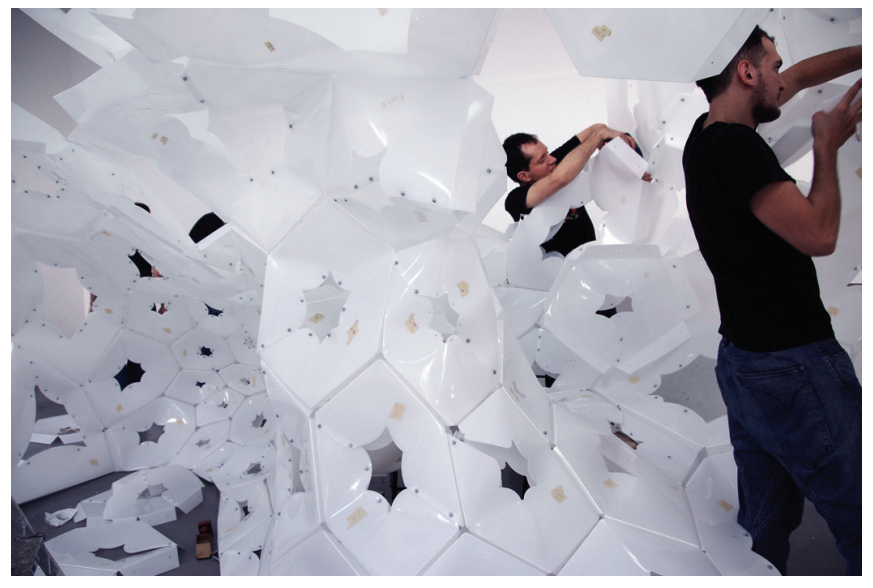

Figure 11: Assembly of the pavilion.

Research papers in photonics revealed that, similar to that of butterflies and certain sea organisms, the beetles' color is generated by its cellular structure as light scatters within its Voronoi texture. Given the program of a display space for video installation, we started to test different lighting effects in relation to the cell geometry. The most promising was an inward orientation of each cellular surface that made it easier to relate different lighting effects to the individual cell. Taking advantage of the polymer's semi-transparent quality, we used LEDs to light the individual cells from inside. 


\section{Conclusion:}

The paper uses the Nuit Blanche pavilion as a case study to demonstrate how the elastic qualities of high density polymers can be used to create a new form of a lightweight structural envelope. In the context of an industry that is changing its production to biopolymers and increasing material recyclability, this paper calls attention to the material's potential to constitute a more dynamic and complex architectural system. The project used Grasshopper and finite element plug-in Karamba to integrate dynamic material behavior, geometry and structure in a generative design process for an exhibition pavilion that could be adapted to a variety of requirements. The project demonstrates how digital design and fabrication techniques can be used to synthesize complex material characteristics with the program of an exhibition space for interactive art installations and the requirements of deployability, structure and envelop.

\section{Acknowledgments}

The author wishes to thank the following contributors to the work described here.

Artist Collaborators: Damien Valero, Jerôme Pougnan, (sound), Jerôme Cognet (video)

Design, Geometry, Fabrication, Assembly: Gernot Riether with Sabri Gökmen and Crimson Changsup Lee

Structural Analysis: Keyan Rahimzadeh
Fabrication: DFL, Georgia Institute of Technology

Special thanks are due also to Andres Cavieres, Matthew Swarts, Ursula Frick, Anthony Payne, Aaron Coffman, Suhee Oh, Kasia Zycinska, Freya Schlemmer.

\section{References}

Williams, J. H. (2008). Employee engagement: Improving participation in safety. Professional Safety, 53(12), 40-45.

Ahlquist, S. and Menges, A. (2011). Behavior-based Computational Design Methodologies: Integrative processes for force defined material structures, ACADIA 2011: Integration through Computation, Banff, 82-89.

Freinkel, S. (2011, March 17). Plastic: Too Good to Throw Away, The New York Times.

LeCuyer, W.A. (2008). ETFE: Technology and Design, Birkhäuser Verlag AG, Basel.

Menges,A. (2011). Integrative Design Computation: Integrating material behavior and robotic manufacturing processes in computational design for performative wood constructions, ACADIA 2011: Integration through Computation, Banff, 2011, 72-81.

Riether, G. (2011). System Interaction, Journal of Green Building, Volume 6, Number 2, 29-35.

Riether, G. and Jolly, K. (2011). Flexible Systems: Flexible Design, Materia and Fabrication: The AIA pavilion as a case study, eCAADe 2011: Respecting Fragile Places, Ljubljana, 2011, 628-634.

Vivek, S. (2009). Structural Origin of CircularlyPolarized Iridescence in Jeweled Beetles, Science, 325, 449-451. 